Research Paper

\title{
PTM-ssMP: A Web Server for Predicting Different Types of Post-translational Modification Sites Using Novel Site-specific Modification Profile
}

\author{
Yu Liu ${ }^{1 *}$, Minghui Wang ${ }^{1,2 \llbracket *}$, Jianing $\mathrm{Xi}^{1}$, Fenglin $\mathrm{Luo}^{1}$ and Ao Li1,2 \\ 1. School of Information Science and Technology, University of Science and Technology of China, Hefei AH230027, China; \\ 2. Centers for Biomedical Engineering, University of Science and Technology of China, Hefei AH230027, China. \\ * These authors contributed equally to this work \\ $\square$ Corresponding author: M.W. (email: mhwang@ustc.edu.cn) \\ (c) Ivyspring International Publisher. This is an open access article distributed under the terms of the Creative Commons Attribution (CC BY-NC) license \\ (https://creativecommons.org/licenses/by-nc/4.0/). See http://ivyspring.com/terms for full terms and conditions.
}

Received: 2017.12.01; Accepted: 2018.01.24; Published: 2018.05.22

\begin{abstract}
Protein post-translational modifications (PTMs) are chemical modifications of a protein after its translation. Owing to its play an important role in deep understanding of various biological processes and the development of effective drugs, PTM site prediction have become a hot topic in bioinformatics. Recently, many online tools are developed to prediction various types of PTM sites, most of which are based on local sequence and some biological information. However, few of existing tools consider the relations between different PTMs for their prediction task. Here, we develop a web server called PTM-ssMP to predict PTM site, which adopts site-specific modification profile (ssMP) to efficiently extract and encode the information of both proximal PTMs and local sequence simultaneously. In PTM-ssMP we provide efficient prediction of multiple types of PTM site including phosphorylation, lysine acetylation, ubiquitination, sumoylation, methylation, O-GaINAc, O-GIcNAc, sulfation and proteolytic cleavage. To assess the performance of PTM-ssMP, a large number of experimentally verified PTM sites are collected from several sources and used to train and test the prediction models. Our results suggest that ssMP consistently contributes to remarkable improvement of prediction performance. In addition, results of independent tests demonstrate that PTM-ssMP compares favorably with other existing tools for different PTM types. PTM-ssMP is implemented as an online web server with user-friendly interface, which is freely available at http://bioinformatics.ustc.edu.cn/PTM-ssMP/index/.
\end{abstract}

Key words: Post-translational modifications, web server, site-specific modification profile, prediction

\section{Introduction}

Protein post-translational modifications (PTMs) are chemical modifications of a protein after its translation, which regulate variety of critical cellular processes such as cell cycle control, DNA repair, signal transduction and protein-protein interactions. [1-3]. Currently, many types of PTMs have been experimentally found and they play a critical role in various biological processes. For example, as a reversible PTM, phosphorylation not only functions significantly in cell signaling but also related to many diseases [4-6]. Moreover, lysine acetylation plays an important role in regulating gene expression and cellular signaling [7]. Ubiquitination is also implicated in the regulation of a variety of cellular processes, such as budding of retroviral virions, regulation of transcription factor activity and receptor endocytosis [8]. Besides these PTMs, there are many studies describing experimental validated PTMs, such as sumoylation, methylation, sulfation, O-linked glycosylation and proteolytic cleavage [9-14], which also affect many aspects of cellular functionalities and relate to various diseases. Therefore, PTM site 
identification in proteomes is important to study and analyze the underlying molecular mechanisms, which also provide useful information for drug discovery [15-17].

Due to the biological importance of protein PTM, many conventional experimental methods have been used to identify potential PTM sites, such as mass spectrometry (MS) and Chip-Chip. Since experimental methods are high-cost and time-consuming, many studies are dedicated to developing efficient and reliable theoretical computation method for the PTM site prediction. Most of computation methods use local sequence information in PTM site prediction because the local sequence of the PTM site is generally conserved [18-20]. For example, a number of methods are proposed to predict phosphorylation sites, such as PhosphoSVM [21], Musite [22], GPS 3.0 [23], KinasePhos 2.0 [24], PPSP [25] and NetPhos [9]. Besides phosphorylation, many bioinformatics tools have been developed to identify other PTM sites. For example, Ubipred [26] and Ubisite [27] are ubiquitination site prediction server which build prediction model using feature extraction method based on the local sequences. In addition, Shao et al. provide a online service called BRABSB [28] for identification of lysine acetylation site. Despite the success achieved by above sequence based methods, using sequence alone may not provide sufficient information for good prediction performance as PTM is a complex process that involves various biological mechanisms [8, 29-31]. Therefore, additional biological information, such as protein-protein interaction (PPI) $[29,30,32]$ and protein structure information [8, 19, 31], has been introduced by different approaches in the task of PTM site prediction. An interesting phenomenon about PTM is that functional associations may exist among proximal PTMs in the protein sequence, i.e., PTM crosstalk [33-37]. It has been shown that phosphorylation can influence and regulate other PTMs such as sumoylation [38, 39] and O-linked glycosylation [40, 41]. Meanwhile, various crosstalks between lysine acetylation and other PTMs including phosphorylation [42-44] and methylation [45-47] have also been validated. For example, previous studies $[44,48]$ report that lysine acetylation inhibits phosphorylation of its upstream +3 position when peptides containing the KXXS motifs. On the other side, some studies [37, 49] show that there are differences in proximal PTM information for different type of PTM sites due to the existence of intrinsic functional PTM crosstalks. Accordingly, we speculate that nearby PTM sites may be helpful to determine a candidate PTM site. More generally, it may be worthy of bringing the information of proximal PTMs into the task of PTM site prediction, and this idea has been supported by our previous work [50] by using information of in situ PTMs that occurs on the same site.

Inspired by aforementioned research, in this study, we develop a web server called PTM-ssMP for PTM site prediction, which adopts site-specific modification profile (ssMP) to efficiently extract and encode the information of both proximal PTMs and local sequence simultaneously. To assess the performance of PTM-ssMP, a large amount of experimentally verified PTM data are collected from several sources and used to train and test the prediction models. Our results suggest that ssMP consistently contributes to remarkable improvement of prediction performance. In addition, independent tests demonstrate that PTM-ssMP compares favorably with other existing tools for different PTM types. For example, the performance of PTM-ssMP obtain more than $10 \%$ improvement compares with other exist tools for lysine acetylation and ubiquitination site prediction. Another advantage of ssMP is that it can be easily applied to various kinds of PTM types, therefore in PTM-ssMP we provide efficient predictions of multiple types of PTM sites including phosphorylation, lysine acetylation, ubiquitination, sumoylation, methylation, sulfation, proteolytic cleavage, O-GalNAc and O-GlcNAc. Therefore, PTM-sSMP is a comprehensive web server and it would provide effective assistance to researchers who are interested in proteome data.

\section{Methods and materials}

\section{Overall framework}

PTM-ssMP is an online web server that can predict nine types of PTM site by using ssMP. There are four main procedures in our work (Figure 1): (i) constructing a valid dataset to train and test the SVM predictive models for multiple PTM types separately, (ii) selecting local sequence containing 10 residues upand downstream of candidate sites, (iii) extracting ssMP for each candidate site (iv) and developing a user-friendly web server called PTM-ssMP that is accessible to the public.

\section{Data collection and pre-processing}

The experimentally determined phosphorylation, lysine acetylation, ubiquitination, sumoylation, methylation, sulfation, proteolytic cleavage, O-GalNAc and O-GlcNAc sites are extracted from several public databases including dbPTM version 3.0 [51], SysPTM [52], Phospho.ELM [53], HPRD [54], PhosphoSitePlus [55] and dbOGAP [56]. We then extract all of the experimentally verified PTM 
sites in human protein from these databases for further analysis. To eliminate sequence redundancy and avoid overestimation of the prediction performance, we use CD-HIT [57] to ensure that none of the protein sequences show a sequence similarity more than $40 \%$ for dataset of each PTM type $[49,58]$. Subsequently, we totally obtain 7866 protein sequences with at least one of the nine PTM types investigated in this study, and experimentally determined PTM sites with proximal PTM information are extracted as positive samples for each PTM type, respectively. We use a local sliding window that comprised 21 residues $[27,59]$ to extract the local sequence and proximal PTM information around candidate sites, where the candidate sites are located at the center with ten neighboring residues upstream and downstream. We find that more than $70 \%$ of the PTM sites have at least one proximal PTM site. For negative samples, since it is difficult to verify that a particular residue is a not PTM site under any conditions [60], we extract negative sites for each PTM type with a common requirement $[60,61]$ that for a specific PTM type, the residue is not verified as a modification site of such PTM type in any database and that protein contains at least one residue known to be modified by such PTM type. To precisely assess the prediction performance, only negative sites with proximal PTMs are used for further analysis. To address a common issue in PTM site prediction that the dataset is unbalanced with much more negative samples than positive ones [21, 62, 63], we follow a widely used procedure by randomly selecting negative samples to match the number of positive ones [21, 62,63] and the final datasets are outlined in Table S1. Furthermore, in consistent with previous studies [61, 64], we randomly select $\sim 20 \%$ sample as the independent test datasets and the remaining part as the benchmark datasets. The numbers of samples in the benchmark datasets and independent test datasets are outlined in Table S2 and Table S3 respectively, and their detailed sequences and positions information in the proteins are given in download page of web server (http://bioinformatics.ustc.edu.cn/PTM-ssMP/download/). Moreover, to reduce the bias of randomness, we follow the previous study [63] to repeat selection procedure of negative sample10 times for benchmark dataset.

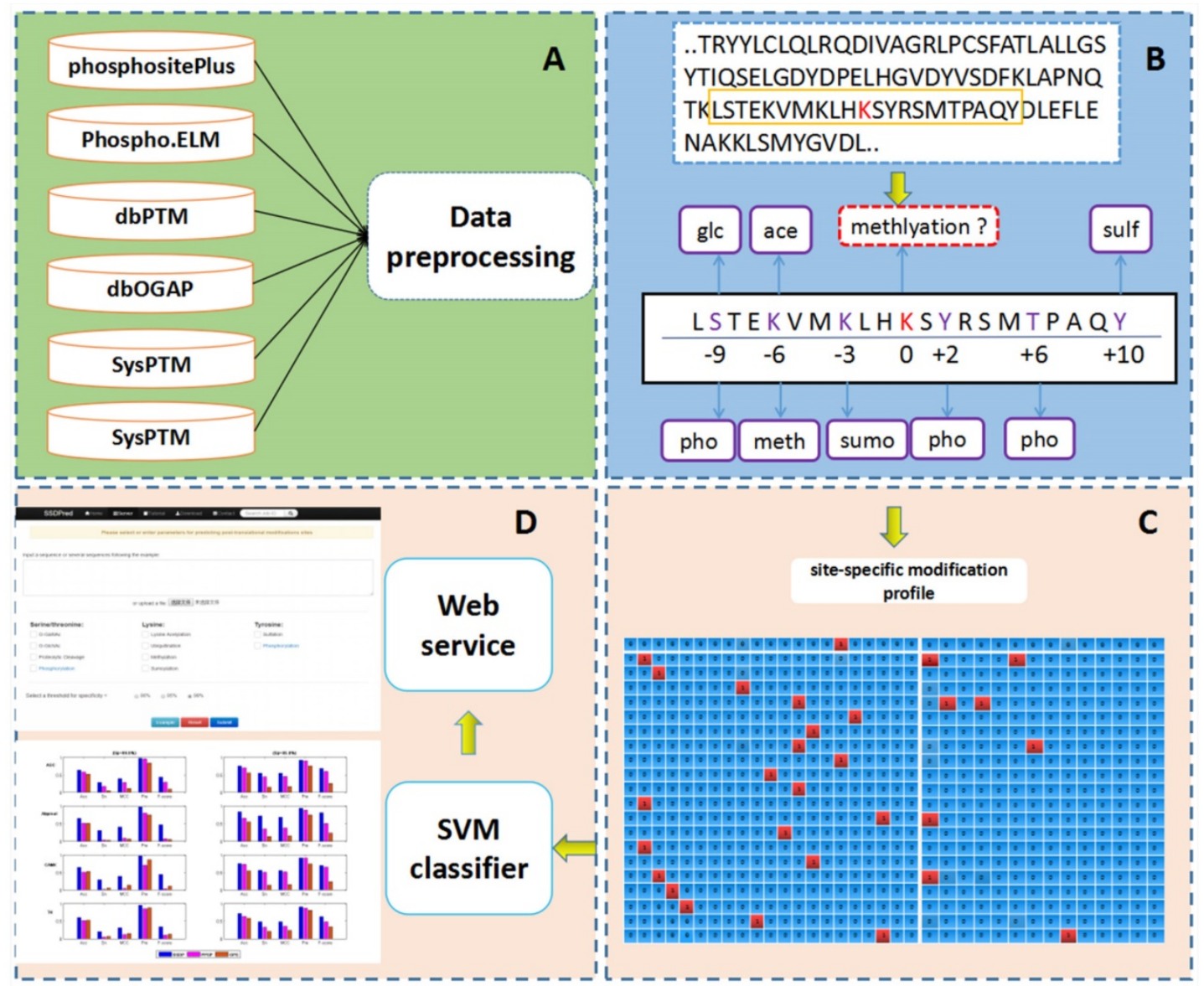

Figure 1. The overall framework of PTM-ssMP 


\section{Construction of site-specific modification profile}

We denote a specific amino acid $R_{0}$ as a valuable of attribute data with $N_{a}$ types of values which represent the $N_{a}$ types of amino acids (here $N_{a}=20$ ), i.e. $R_{0} \in S_{a}=\left\{A_{j} \mid j=1, \ldots, N_{a}\right\}$ where $A_{j}$ is the $j$-th type of amino acid. To predict the PTM types on residue $R_{0}$, we define $R_{i}$ as the $i$-th downstream amino acid of the $R_{0}$. The set $\left\{R_{i} \mid 1 \leq i \leq L, i \in \mathrm{N}\right\}$ is the $L$-th order downstream residue set, which contains the downstream amino acid residue neighbors of $R o$. Similarly, $R_{-i}$ denotes the $i$-th upstream amino acid of the $R_{0}$, and the set $\left\{R_{-i} \mid 1 \leq i \leq L, i \in N\right\}$ is the $L$-th order upstream residues set of $R$ o. We then define the local sequences of $R_{0}$ as a set $S_{e q}$, i.e.

$$
S e q_{L}=\left\{R_{-i} \mid-L \leq i \leq L, i \in N\right\}
$$

where the cardinality of $P_{L}$ is $2 L+1$ and $L$ is set to 10 in this study. Specifically, when the length of the upstream sequence $L_{u}$ or the length of the downstream sequence $L_{d}$ is less than $L$, we use '*' to represent the not-exist amino acid, and the values of these not-exist amino acid is the dummy attribute. We further define another attribute variable $Q_{i}$ to denote known PTM types on $R_{i}$ that have been previously validated, of which the value represents the $N_{P}$ types of PTMs. The variable space of $Q_{i}$ is a set whose cardinality is $N_{P}$ (here $\left.N_{P}=14\right)$, which is defined as $S_{P}$ $=\left\{P_{j} \mid j=1, \ldots, N_{P}\right\}$ and $P_{j}$ is the $j$-th type of PTM.

To encode the information of proximal PTMs, we introduce a $N_{P} \times(2 L+1)$ dimension matrix $Y_{i j}$ to represent the profile of proximal PTMs for the sites where $-L \leq i \leq L$. Here $N_{P}$ represents the totally number of PTM types for attribute variable $Q_{i}$. The $(\mathrm{i}, \mathrm{j})$ -th element of the matrix $Y_{i j}$ is:

$Y_{i j}=\operatorname{Ind}\left(Q_{i}=P_{j}\right)$, for $-L \leq i \leq L$ and $1 \leq j \leq N_{P T M}, i, j \in N$

where $P_{j}$ is $j$-th type of PTM that is represented by the $j$-th feature of the vector $Y_{i}$. The function Ind $(\cdot)$ is the indicator function, which gives an output of 1 when the input equation is true and 0 otherwise. Since there may be more than one type of known PTM occurring on a same site, the matrix $Y_{i j}$ for representing known PTM types is not orthogonal. For example, there is a local sequence in Figure 1B, which consist of 21 amino acids (including a candidate site at the center and its local sequence at both upstream and downstream). In this local sequence, acetylation and methylation occurred on upstream 6 position, correspondingly, these PTM information are represented as $(0,1,0,1,0,0,0,0,0,0,0,0,0,0)$. Likewise, we encode the local sequence information as a $N_{R} \times$ $(2 L+1)$ dimension matrix $\left(X_{i j}\right)$, which is also the numeric representation of $R_{i}$ for $-L \leq i \leq L$. The entry $x_{i j}$ of the matrix is calculated as

$$
X_{i j}=\operatorname{Ind}\left(R_{i}=A_{j}\right) \text {, for }-L \leq i \leq L \text { and } 1 \leq j \leq N_{R}, i, j \in \mathrm{N}
$$

where $A_{j}$ is the $j$-th type of amino acid in set $S_{R}$ that is represented by the $j$-th feature of the vector. Note that $X_{i j}$ is an orthogonal binary matrix with only one entry of each row assigned with 1 and others assigned with 0 . For example, a local sequence like 'LSTEKVMKLHKSYRSMTPAQY', which will be encoded into a 21 by 21 matrix. Specifically, the first site of this local sequence is ' $\mathrm{L}$ ', and it will be encoded as $\quad(0,0,0,0,0,0,0,0,0,0,0,0,0,0,0,1,0,0,0,0,0)$, correspondingly, the first row of the matrix is $(0,0,0,0,0,0,0,0,0,0,0,0,0,0,0,1,0,0,0,0,0)$.

Finally, we define the ssMP matrix $Z_{i j}$ as $Z_{i j}=X_{i j}$ $\oplus Y_{i j}$, where the symbol $\oplus$ is the concatenation operation of matrices $X_{i j}$ and $Y_{i j}$. The variable space is the Cartesian product of the spaces for the two variables $S_{Z}=S_{R} \otimes S_{P}$ (the symbol $\otimes$ denotes to Cartesian product). For all the entries $Z_{i j}$ in the ssMP matrix, we can extract the features contained in $S_{Z}$ of the sample for each site in the local sequence and the related PTMs. To develop the classification models for PTM site prediction, a ssMP is generated for each sample in the training data and the entries of the ssMP are then adopted as the input features to train a SVM classifier with LibSVM [65] for each PTM type. These classifiers are then used to make perditions with the ssMP features extracted from candidate PTM sites.

\section{Implementation and web interface}

PTM-ssMP is implemented by the python on a Linux machine with Apache 2.2.3 HTTP Server. Web server consists of six sections, including Home, Server, Tutorial, Result, Download and Contact. Home page include a brief introduction about PTM-ssMP. In Server page (Figure 2), users can submit the data of proteins with known PTM information and select the task-related prediction models and thresholds. For the convenience of most users, we present a user guide in Tutorial page and our contact information in contact page. Furthermore, PTM-ssMP allows users to download datasets of all PTM types, including corresponding Protein ID, Position and protein local sequences from Download page. After the prediction task, PTM-ssMP generates a Result page that contains prediction results, where each row of the result represents a candidate site. The running time required for a prediction task depends on the selected models and the length of the query sequence. In PTM-ssMP we provide a test example, which have two query sequences with the total length more than 500 amino acid. The prediction task requires $10 \mathrm{~s}$ to generate and return the results if select 
all predictive models.

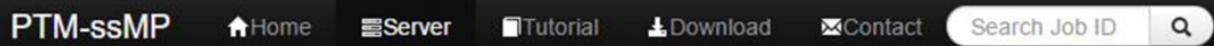

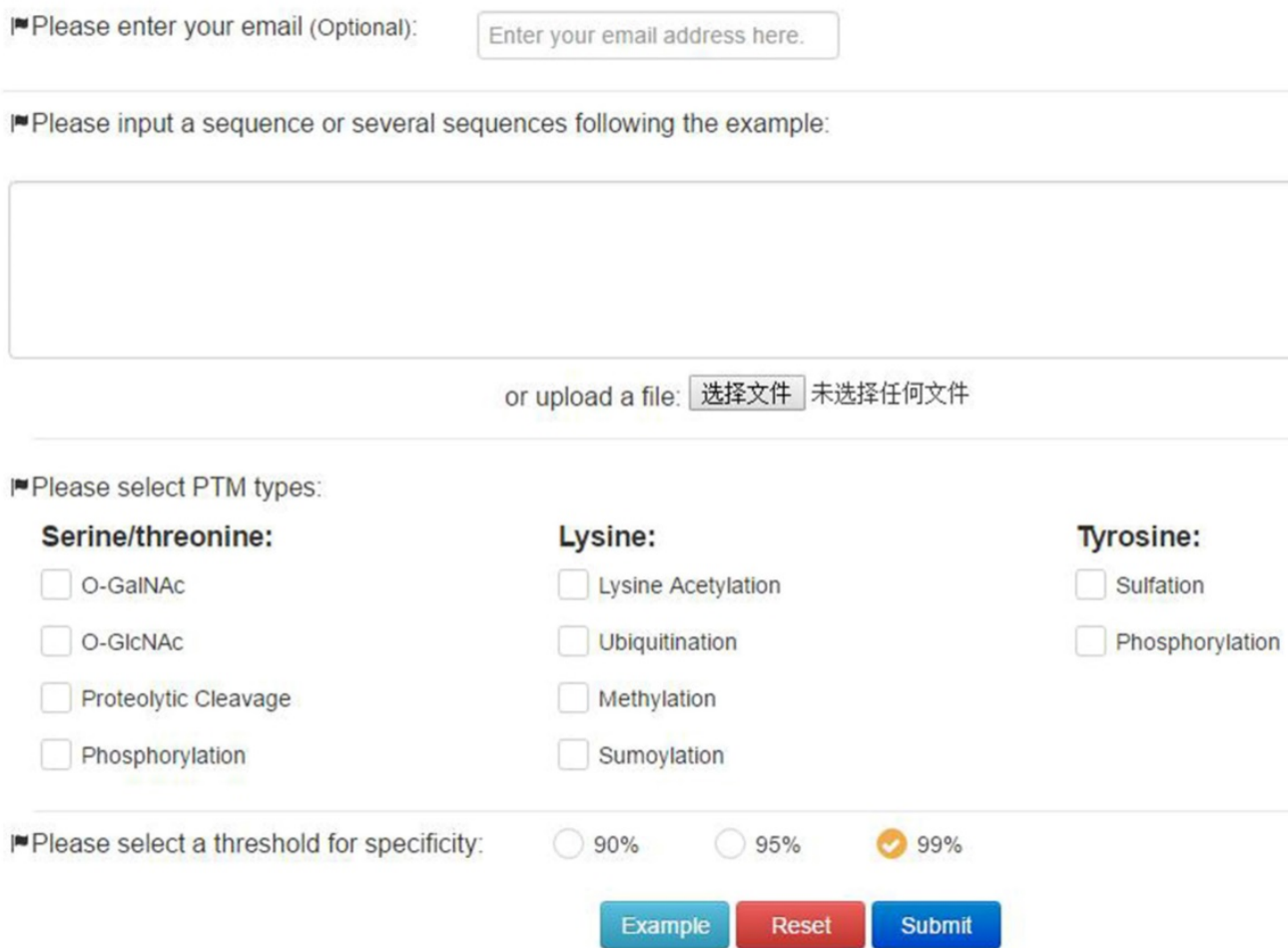

Figure 2. A semi-screenshot to show the server-page of the PTM-ssMP web-server at http://bioinformatics.ustc.edu.cn/PTM-ssMP/server/

\section{Performance evaluation}

In this study, by following previous studies we perform a 10-fold cross-validation [2, 25] (based on the benchmark datasets) to evaluate the importance of ssMP and independent tests [27, 61](based on the independent test datasets) to compare with other existing PTM prediction tools. To evaluate the performance of each prediction model, six standard measurements are adopted, including accuracy $(A c c)$, specificity $(S p)$, sensitivity $(S n)$, Matthew's correlation coefficient (MCC), precision (Pre) and F1 score (F1). They are defined as follows:

$$
\begin{gathered}
\mathrm{Acc}=(\mathrm{TN}+\mathrm{TP}) /(\mathrm{TN}+\mathrm{TP}+\mathrm{FN}+\mathrm{FP}) \\
\mathrm{Sn}=\mathrm{TP} /(\mathrm{TP}+\mathrm{FN}) \\
\mathrm{Sp}=\mathrm{TN} /(\mathrm{TN}+\mathrm{FP}) \\
\mathrm{Pre}=\mathrm{TP} /(\mathrm{TP}+\mathrm{FP}) \\
\mathrm{F} 1=2 \times \mathrm{Pr} \times \mathrm{Sn} /(\mathrm{Pre}-\mathrm{Sn}) \\
\mathrm{MCC}=(\mathrm{TP} \times \mathrm{TN}-\mathrm{FP} \times \mathrm{FN}) /[(\mathrm{TP}+\mathrm{FN}) \times(\mathrm{TP}+\mathrm{FP}) \\
\times(\mathrm{TN}+\mathrm{FN}) \times(\mathrm{TN}+\mathrm{FP})]^{1 / 2}
\end{gathered}
$$

$T P$ and TN are abbreviations of true positives and true negatives, which indicate the number of positive and negative sites that are correct predicted. $F P$ and $F N$ are abbreviations false positives and false negatives, which indicate the number of positive and negative sites that are predicted falsely. $S n$ and $S p$ are abbreviations of sensitivity and specificity, and they measure the proportion of positives and negatives that are correctly identified. MCC is used to reflect the balance quality when the numbers of negative and positive data are significant imbalance.

\section{Results}

\section{Statistical difference of proximal PTMs in positive and negative samples}

We first investigate the statistical difference of proximal PTMs in positive and negative samples. Use lysine acetylation as example, previous studies [44, 48 report that lysine acetylation inhibits phosphorylation in +3 positions. Consequently, we calculate the number of phosphorylation of the +3 positions in ssMP of positive and negative samples respectively, and the statistical significance is examined by using Fisher exact test. The results (Figure 3A) show that phosphorylation in +3 positions of acetylation-modified lysine is lower $(p=7.73 \times$ $\left.10^{-7}\right)$, which indicate that ssMP can reflect the proximal PTMs difference between positive and negative samples and it could be a helpful feature for 
PTM site prediction. Furthermore, many studies report that there is mutual exclusion between lysine acetylation and its upstream phosphorylation. [66-68]. Correspondingly, we perform the same analysis on all upstream phosphorylation between lysine acetylation positive and negative samples, and we find a significant difference between the numbers of upstream phosphorylation in positive and negative samples $\left(p=4.05 \times 10^{-44}\right)$ (Figure 3B). The above analysis shows that there are clear differences between the proximal PTMs of positive and negative samples, which might contribute to the PTM site prediction.
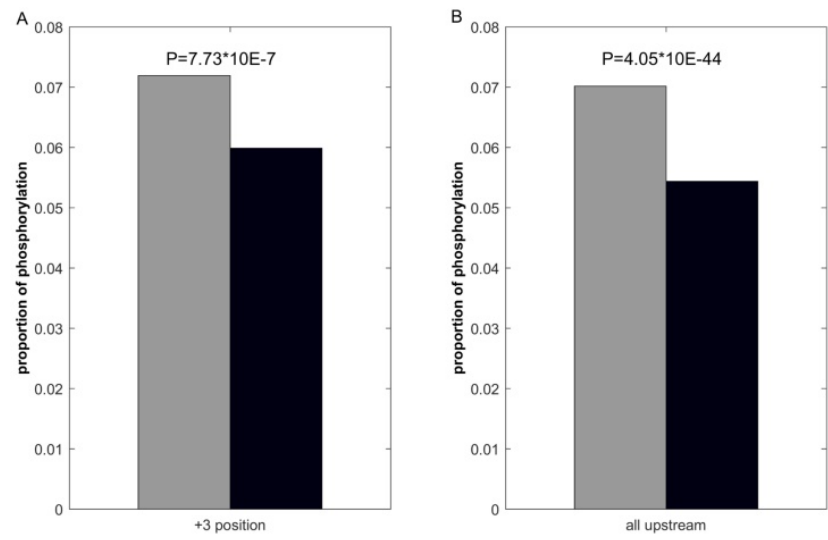

Figure 3. Comparison of proximal PTMs between positive and negative data. The gray bar represents the proportion of lysine acetylation proximal phosphorylation of positive samples and the black bar represents the negative ones.

\section{Evaluation of the prediction model}

In this section, we adopt ten-fold cross-validation to evaluate the performance of ssMP in PTM site prediction based on benchmark dataset, and we assess the prediction models trained by both local sequences and ssMP are assessed. In Figure S1 we show the ROC curves of ubiquitination, acetylation, O-GlcNAc and phosphorylation in AGC, CK1, STE kinase groups. Furthermore, in Table 1 we list the corresponding AUC values in terms of mean and variance. As can be seen from Figure S1, our proposed method that are trained with ssMP has better prediction performance in predicting multiple types PTM sites. For phosphorylation kinase group AGC, STE and CK1 the AUC values of method trained with only local sequences are $0.822,0.728$ and 0.852 , respectively. In our proposed method, the AUC values of AGC, STE and CK1 are increased to 0.873, 0.806 and 0.901 . In addition, for ubiquitination, the AUC value of method trained with local sequences only is 0.668 . With ssMP, the corresponding AUC value is 0.752 , which is $8.4 \%$ higher than the method trained with local sequences. Similarly, for lysine acetylation, and O-GlcNAc, the AUC value of our proposed method with ssMP also obtain $9.5 \%, 5.8 \%$ improvement than those methods that only use local sequences. The AUC value of the other PTM types are listed in Table S4, which further suggests that our proposed method is superior to the method that only consider local sequences. Therefore, we believe that ssMP are very useful features which can significant improve the performance of PTM site prediction for multiple types.

Table 1: AUC values comparison of ssMP and local sequences of ubiquitination, lysine acetylation, O-GlcNAc and phosphorylation kinase groups AGC, CK1 and STE.

\begin{tabular}{|c|c|c|c|c|c|}
\hline PTM & method & AUC & PTM & method & AUC \\
\hline $\begin{array}{l}\text { Phosphoryla } \\
\text { tion } \\
\text { (AGC) }\end{array}$ & $\begin{array}{l}\text { SEQ } \\
\text { ssMP }\end{array}$ & $\begin{array}{l}0.822 \pm 1.96 e-5 \\
0.873 \pm 2.76 e-5\end{array}$ & $\begin{array}{l}\text { Ubiquitinati } \\
\text { on }\end{array}$ & $\begin{array}{l}\text { SEQ } \\
\text { ssMP }\end{array}$ & $\begin{array}{l}0.668 \pm 3.00 \mathrm{e}-6 \\
0.752 \pm 1.90 \mathrm{e}-6\end{array}$ \\
\hline $\begin{array}{l}\text { Phosphoryla } \\
\text { tion } \\
\text { (CK1) }\end{array}$ & $\begin{array}{l}\text { SEQ } \\
\text { ssMP }\end{array}$ & $\begin{array}{l}0.852 \pm 7.49 \mathrm{e}-5 \\
0.901 \pm 4.70 \mathrm{e}-5\end{array}$ & $\begin{array}{l}\text { Lysine } \\
\text { acetylation }\end{array}$ & $\begin{array}{l}\text { SEQ } \\
\text { ssMP }\end{array}$ & $\begin{array}{l}0.649 \pm 1.32 \mathrm{e}-5 \\
0.744 \pm 7.14 \mathrm{e}-6\end{array}$ \\
\hline $\begin{array}{l}\text { Phosphoryla } \\
\text { tion } \\
\text { (STE) }\end{array}$ & $\begin{array}{l}\text { SEQ } \\
\text { ssMP }\end{array}$ & $\begin{array}{l}0.728 \pm 3.19 e-4 \\
0.806 \pm 3.33 e-4\end{array}$ & O-GlcNAc & $\begin{array}{l}\text { SEQ } \\
\text { ssMP }\end{array}$ & $\begin{array}{l}0.772 \pm 1.98 \mathrm{e}-4 \\
0.830 \pm 6.12 \mathrm{e}-5\end{array}$ \\
\hline
\end{tabular}

In addition to the AUC value, we also adopt other six measurements including Sn, Sp, Acc, F1, Pre and MCC to verify the reliability of ssMP. By following the study of Wang et al. [69], we set the threshold of specificity equal to $95.0 \%$ (high stringency levels) or $90.0 \%$ (medium stringency levels). Take two phosphorylation kinase groups AGC, STE and another two PTM ubiquitination, lysine acetylation as examples, the corresponding measurements in high stringency levels are computed and reported in Table S3. By incorporating ssMP, the values of Acc, Sn, F1, Pre and MCC for AGC are 0.743, $0.536,0.675,0.915$ and 0.534 , respectively, whereas the Acc, Sn, F1, Pre and MCC values with only local sequences are 0.706, 0.461, 0.610, 0.903 and 0.472, respectively. And for STE, the corresponding measurements are also improved by $7.0 \%, 14.0 \%$, $15.9 \%, 6.0 \%$ and $13.3 \%$, respectively. In addition to phosphorylation, we can find that our proposed method also have better performance for ubiquitination and acetylation. Taken ubiquitination as instance, the values of $A c c, S n, F 1$, Pre and MCC values are $0.588,0.227,0.355,0.819$ and 0.256 , which are increased by $6.2 \%, 12.4 \%, 17.7 \%, 14.7 \%$ and $15.7 \%$ compared with the method using only local sequences. Similarly, as shown in Table S5, for other PTM types and phosphorylation groups our proposed method is consistently better than the method that using only local sequences. We also compute such measurements at medium stringency level, the detailed results of all PTM and phosphorylation groups are listed in Table S6. These results suggest 
that ssMP are efficient features which can significant improve the prediction of multiple PTMs.

\section{Comparison with existing tools}

In this section, base on independent dataset we compare the prediction performance of PTM-ssMP and several other existing tools. Firstly, two common and efficient phosphorylation prediction methods, PPSP and GPS 3.0 are used to make comparison. To illustrate the prediction performance, we take four kinase groups Atypical, CK1, AGC and TK as examples and plot the ROC curves for three methods (Figure 4). As shown in Figure 4, for four kinase groups PTM-ssMP achieves better performance than other prediction methods. Moreover, for each method we also calculate the corresponding AUC value for each phosphorylation kinase group and displayed in Figure 4. For Atypical, CK1, AGC and TK, the AUC values of PTM-ssMP are 0.924, 0.880, 0.894 and 0.821 , which is $10.8 \%, 25.5 \%, 23.1 \%$ and $8.4 \%$ higher than GPS, and the AUC value of PPSP only have 0.813, $0.808,0.833$ and 0.765 , respectively. Therefore, PTM-ssMP outperformed other prediction methods in predicting multiple kinase groups of phosphorylation. Meanwhile, the ROC curves for other kinase groups are also plotted (Figure S3), and we find that PTM-ssMP consistently better than other
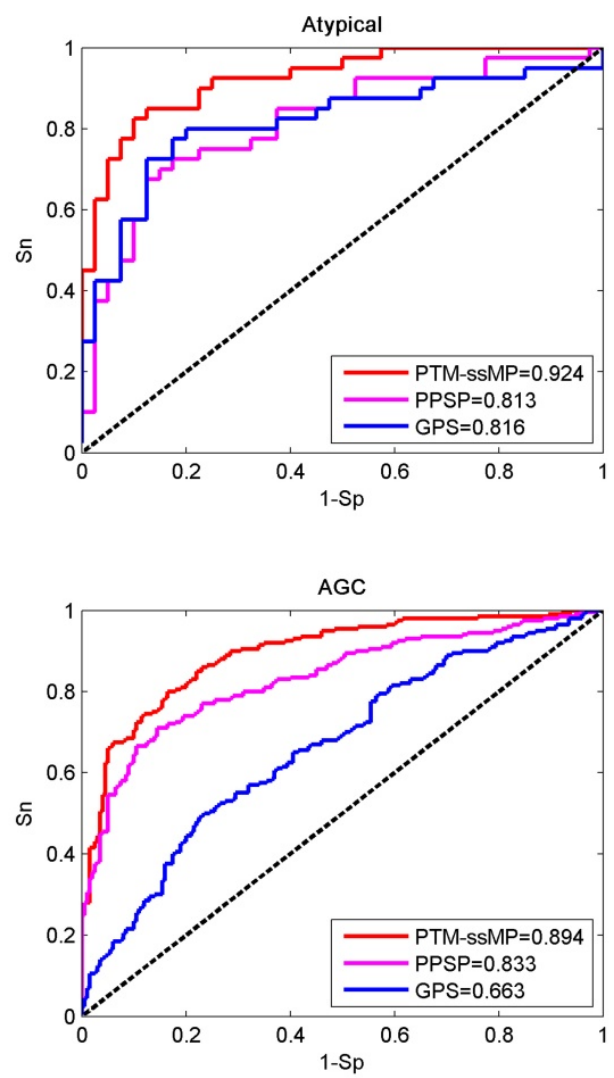

methods.

Additionally, we also plot the Acc-Sn-MCCPre-F1 bar graph of three methods to assess the detailed performance for phosphorylation kinase group AGC, CK1, STE and Atypical according to the high and medium stringency levels, as shown in Figure 5. It is obvious that PTM-ssMP achieves the best prediction performance in most of kinase groups. For instance, at high stringency level ( $S p=95.0 \%)$, the Acc, Sn, MCC, Pre, F1 values of AGC are increased by 8.5\%, 17.0\%, 14.2\%, 2.5\%, 14.1\% compared with PPSP. For STE, the Acc, Sn, MCC, Pre, F1 values are improved by $15.0 \%, 30.0 \%, 27.6 \%, 11.1 \%, 30.8 \%$ compared with PPSP. Similarly, PTM-ssMP has better or comparable performance than GPS in most of kinase groups. In addition, PTM-ssMP also obtain better performance at medium stringency $(S p=90 \%)$. Taking CK1 as an example, PTM-ssMP outperforms PPSP with 5.5\%, 11.0\%, 9.8\%, 1.8\%, 7.6\% higher Acc, Sn, MCC, Pre, F1 values. The detailed results other groups at high stringency level and medium stringency level are listed in Table S7 and Table S8 respectively. As can be seen from the results, PTM-ssMP has better performance than other prediction method in almost all kinase groups.
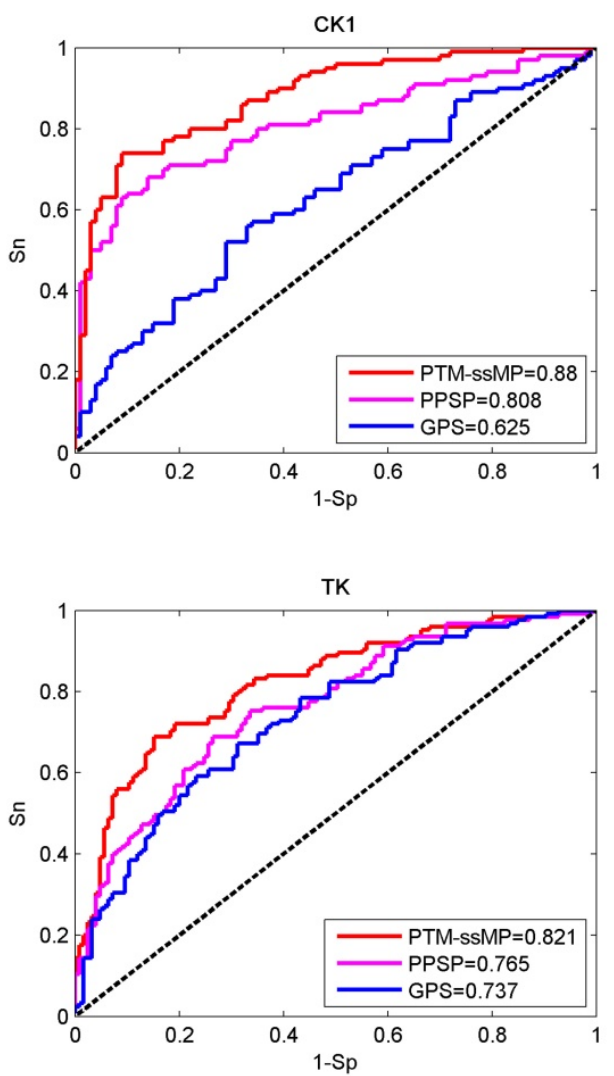

Figure 4. Performance of phosphorylation ROC curves in kinase group Atypical CK1, AGC and TK with different methods. The red lines represent the performance of PTM-ssMP, the blue and purple lines represent the GPS, PPSP respectively. 

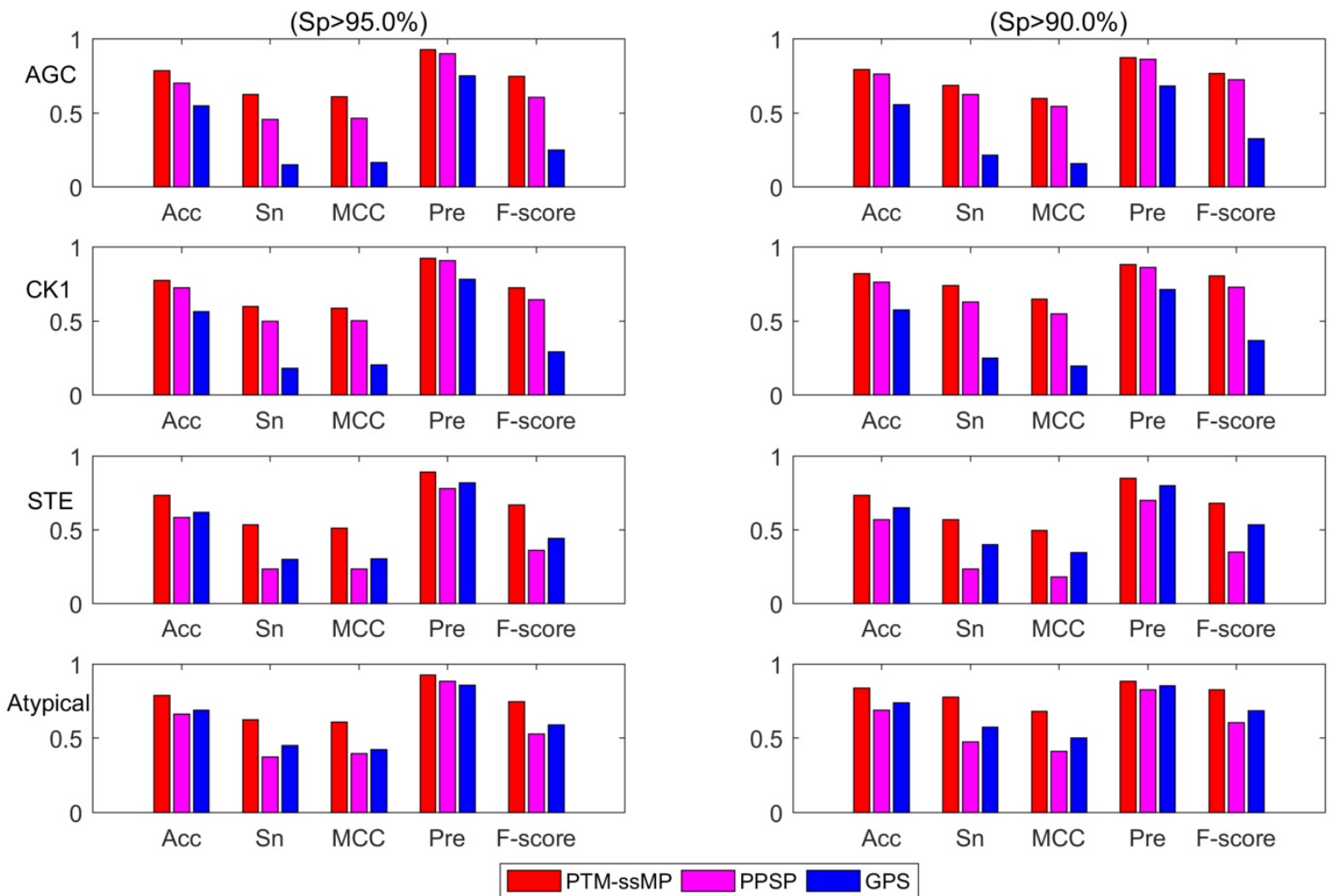

Figure 5. The Acc, Sn, MCC, Pre, F-score value comparison with different methods for kinase group AGC, CK1, STE and Atypical at two stringency levels. The left part is at specificity of $95.0 \%$, the measurements in the right one are at specificity of $90.0 \%$. The horizontal axis represents accuracy, sensitivity, Matthew correlation coefficient, precision and F-score respectively.

In order to assess the prediction performance of PTM-ssMP more comprehensively, besides phosphorylation, we compare PTM-ssMP with other PTM type prediction tools. For ubiquitination, we compare PTM-ssMP with two common ubiquitination prediction methods Ubipred [26] and Ubisite [27], and the ROC curves of this methods are plotted and shown in Figure S4A. PTM-ssMP achieves AUC value of $75.0 \%$, and the corresponding AUC values of Ubisite and Ubipred are $58.4 \%$ and $54.2 \%$ (Figure S4A). For lysine acetylation, PAIL [70] and BRABSB-PHKA [28] are applied to compare the prediction performance. As shown in Figure S4B, the AUC values are increased by $15.4 \%$ compared with BRABSB-PHKA and $21.8 \%$ with PAIL.

As we all known, the prediction top-ranked results are very important in practice, which is used for proteomic-wide screening and systematic examination [71]. This requires computational method with both low false positive rate and ability to predict potential PTM site [71, 72]. Hence, we follow previous studies $[71,73]$ to compare the number of correctly retrieved PTM sites in top-ranked results. For each percentile $\mathrm{k} \%$, we count the number of true PTM site in the top ranked $\mathrm{k} \%$ *total samples predictions and their proportion. Here, we take lysine acetylation and ubiquitination as example, results of six percentiles $1 \%, 2 \%, 5 \%, 10 \%, 15 \%$ and $20 \%$ of the total corresponding PTM sites number are compared, as shown in Figure 6. It is observed that, for ubiquitination, almost at all percentiles PTM-ssMP have more true positive prediction than Ubisite and Ubipred, and for lysine acetylation, our method also consistently better than BRABSB-PHKA and PAIL. In conclusion, aforementioned analyses suggest that PTM-ssMP obtain better performance than the other prediction tools in predicting multiple types of PTM.

\section{Usage}

For the convenience of most users, a detailed user guide is provided below.

(1) Opening the web-server of PTM-ssMP at http:/ / bioinformatics.ustc.edu.cn/PTM-ssMP/server /, users can see the server page of PTM-ssMP on their computer screen. (2) On the server page, users can input the query protein sequences into the input box at the center of Figure 2 or choose the batch prediction by upload they desired batch input file via the 'Browse' button. PTM-ssMP requires FASTA format inputs, users can click the 'Example' button left below 
the input box to obtain the examples of sequences with FASTA format. (3) Select PTM type and specificity threshold, click on the 'Submit' button to submit the prediction task. (4) After the prediction task, PTM-ssMP will generate a result page that contains prediction results, each row of the result

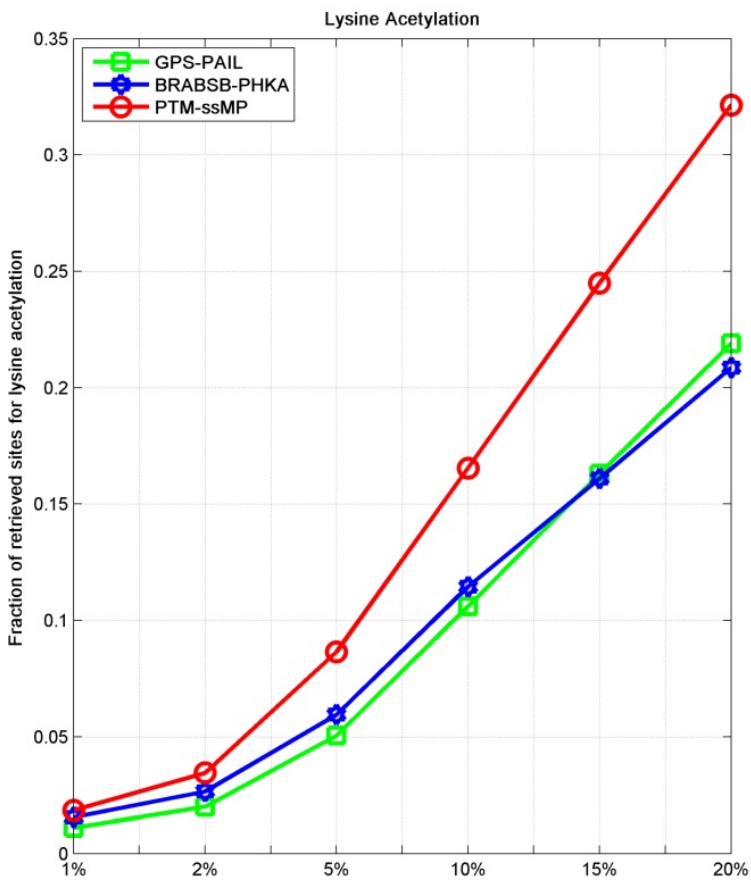

represents a candidate site (Figure 7). Users can export prediction result in JSON, TXT, XML, CSV, SQL and MS-EXCEL format by click 'export data' button. (5) Users can click the Download button at the top of the page to download all types of PTM datasets that is used to train and test the current predictors.

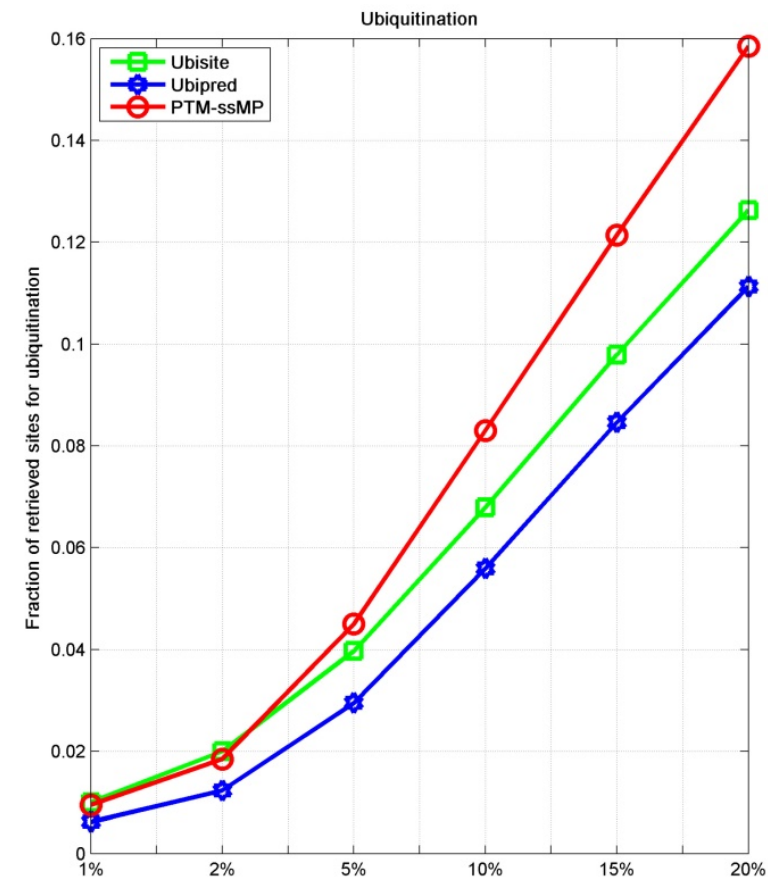

Figure 6.The fraction of retrieved sites for lysine acetylation and ubiquitination (A) The left part represents the performance of Lysine Acetylation, and (B) the right part represents the performance of Ubiquitination. The horizontal axis represents five top 1, 2, 5, 10,15 and 20 percent of the total samples.

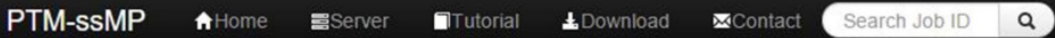

\section{"Parameters:}

PTM types: Lysine Acetylation, Ubiquitination,Methylation, Sumoylation

thresholds: $90.0 \%$

\section{"Results:}

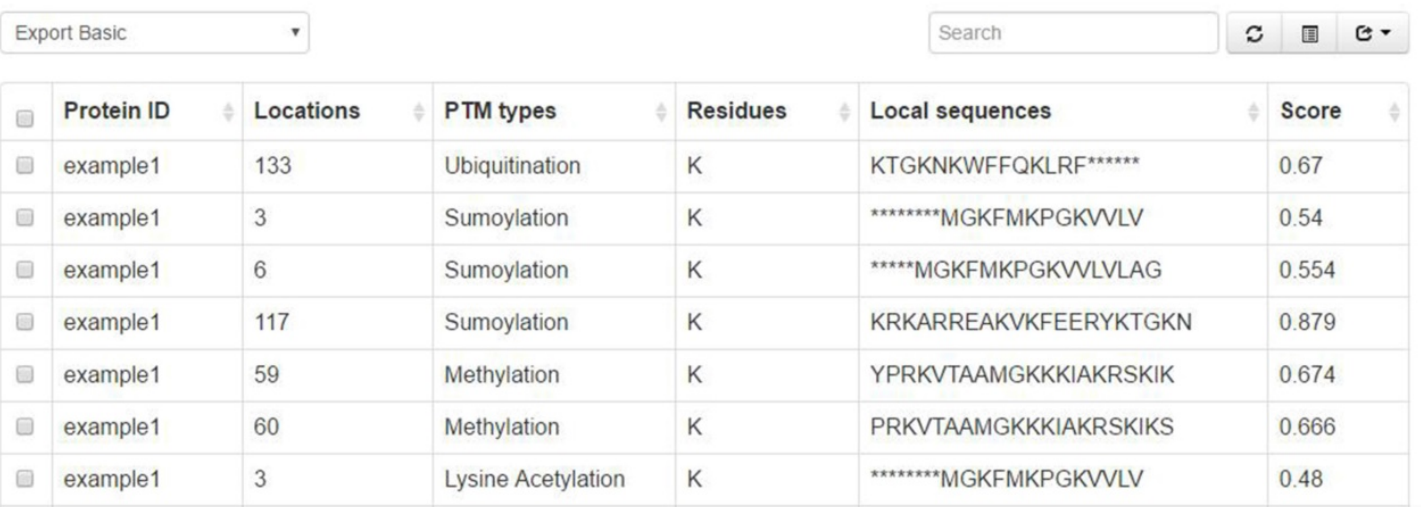

Figure 7. A semi-screenshot to show an example of PTM-ssMP prediction result 


\section{Conclusions}

PTMs play a critical role in various cellular processes, including maintain protein structure and integrity, regulate metabolism and defense processes. However, experimental methods are high-cost and time-consuming, it is urgent to develop effective tools to predict PTM site. Many PTM prediction tools only adopt the local sequence information or functional information for candidate site without considering the relations between different PTMs, which may limit the prediction performance. In this work, we develop a novel web server called PTM-ssMP to predict multiple PTM sites, which adopts the ssMP that can efficiently incorporate the relationships between substrate sites and PTMs. PTM-ssMP allows users submit multiple query protein sequences simultaneously and export prediction result in a variety of format. In addition, as can be seen from the results, the performance of PTM-ssMP is better than other existing tools. Overall, we believe PTM-ssMP will be very helpful for the identification of multiple types of PTM site.

\section{Supplementary Material}

Supplementary figures and tables. http://www.ijbs.com/v14p0946s1.pdf

\section{Acknowledgements}

This work is supported by National Natural Science Foundation of China (No. 61471331, No. 61571414 and No. 61101061), University of Science and Technology of China, USTC. We appreciate the valuable suggestions from any reviewers.

\section{Author contributions}

All of the authors listed made substantial contributions to the manuscript and qualify for authorship, and no authors have been omitted. Conception and design: $\mathrm{Yu}$ Liu; development of methodology and acquisition of data: $\mathrm{Yu} \mathrm{Liu}$, Minghui Wang, Fenglin Luo, Ao Li; analysis and interpretation of data: Yu Liu, Jianing Xi, Fenglin Luo, Ao Li; writing and revision of the manuscript: $\mathrm{Yu} \mathrm{Liu,}$ Jianing Xi, Minghui Wang, Fenglin Luo Ao Li.

\section{Competing Interests}

The authors have declared that no competing interest exists.

\section{References}

1. Song C, Ye M, Liu Z, Cheng H, Jiang X, Han G, et al. Systematic analysis of protein phosphorylation networks from phosphoproteomic data. Molecular \& Cellular Proteomics. 2012; 11: 1070-83.
2. Xu X, Li A, Zou L, Shen $Y$, Fan W, Wang M. Improving the performance of protein kinase identification via high dimensional protein-protein interactions and substrate structure data. Molecular bioSystems. 2014; 10: 694-702.

3. Zhu L, Li N. Quantitation, networking, and function of protein phosphorylation in plant cell. Frontiers in plant science. 2012; 3:302.

4. Ubersax JA, Ferrell Jr JE. Mechanisms of specificity in protein phosphorylation. Nature reviews Molecular cell biology. 2007; 8: 530-41.

5. Matthews HR. Protein kinases and phosphatases that act on histidine, lysine, or arginine residues in eukaryotic proteins: a possible regulator of the mitogen-activated protein kinase cascade. Pharmacology \& therapeutics. 1995; 67: 323-50.

6. Li L, Shakhnovich EI, Mirny LA. Amino acids determining enzyme-substrate specificity in prokaryotic and eukaryotic protein kinases. Proceedings of the National Academy of Sciences. 2003; 100: 4463-8.

7. Zhao S, Xu W, Jiang W, Yu W, Lin Y, Zhang T, et al. Regulation of cellular metabolism by protein lysine acetylation. Science. 2010; 327: 1000-4.

8. Radivojac P, Vacic V, Haynes C, Cocklin RR, Mohan A, Heyen JW, et al. Identification, analysis, and prediction of protein ubiquitination sites. Proteins: Structure, Function, and Bioinformatics. 2010; 78: 365-80.

9. Blom N, Sicheritz-Pontén T, Gupta R, Gammeltoft S, Brunak S. Prediction of post-translational glycosylation and phosphorylation of proteins from the amino acid sequence. Proteomics. 2004; 4: 1633-49.

10. Hortin G, Folz R, Gordon JI, Strauss AW. Characterization of sites of tyrosine sulfation in proteins and criteria for predicting their occurrence. Biochemical and biophysical research communications. 1986; 141: 326-33.

11. Shen Z, Pardington-Purtymun PE, Comeaux JC, Moyzis RK, Chen DJ. UBL1, a human ubiquitin-like protein associating with human RAD51/RAD52 proteins. Genomics. 1996; 36: 271-9.

12. Clarke S. Protein methylation. Current opinion in cell biology. 1993; 5: 977-83

13. Xue Y, Zhou F, Fu C, Xu Y, Yao X. SUMOsp: a web server for sumoylation site prediction. Nucleic acids research. 2006; 34: W254-W7.

14. Zhou F, Olman V, Xu Y. Large-scale analyses of glycosylation in cellulases. Genomics, proteomics \& bioinformatics. 2009; 7: 194-9.

15. Gao Y, Hao W, Gu J, Liu D, Fan C, Chen Z, et al. PredPhos: an ensemble framework for structure-based prediction of phosphorylation sites. Journal of Biological Research-Thessaloniki. 2016; 23: 12.

16. Krueger KE, Srivastava S. Posttranslational protein modifications current implications for cancer detection, prevention, and therapeutics. Molecular \& Cellular Proteomics. 2006; 5: 1799-810.

17. Audagnotto M, Dal Peraro M. Protein post-translational modifications: In silico prediction tools and molecular modeling. Computational and structural biotechnology journal. 2017; 15: 307-19.

18. Eisenhaber B, Eisenhaber F. Prediction of posttranslational modification of proteins from their amino acid sequence. Methods in molecular biology. 2010; 609: 365-84.

19. Blom N, Gammeltoft S, Brunak S. Sequence and structure-based prediction of eukaryotic protein phosphorylation sites. Journal of molecular biology. 1999; 294: 1351-62.

20. Miller ML, Blom N. Kinase-specific prediction of protein phosphorylation sites. Phospho-Proteomics: Methods and Protocols. 2009: 299-310.

21. Dou Y, Yao B, Zhang C. PhosphoSVM: prediction of phosphorylation sites by integrating various protein sequence attributes with a support vector machine. Amino acids. 2014; 46: 1459-69.

22. Gao J, Thelen JJ, Dunker AK, Xu D. Musite, a tool for global prediction of general and kinase-specific phosphorylation sites. Molecular \& Cellular Proteomics. 2010; 9: 2586-600.

23. Xue Y, Ren J, Gao X, Jin C, Wen L, Yao X. GPS 2.0, a tool to predict kinase-specific phosphorylation sites in hierarchy. Molecular \& cellular proteomics. 2008; 7: 1598-608

24. Wong Y-H, Lee T-Y, Liang H-K, Huang C-M, Wang T-Y, Yang Y-H, et al. KinasePhos 2.0: a web server for identifying protein kinase-specific phosphorylation sites based on sequences and coupling patterns. Nucleic acids research. 2007; 35: W588-W94

25. Xue Y, Li A, Wang L, Feng H, Yao X. PPSP: prediction of PK-specific phosphorylation site with Bayesian decision theory. BMC bioinformatics. 2006; 7: 163

26. Tung C-W, Ho S-Y. Computational identification of ubiquitylation sites from protein sequences. BMC bioinformatics. 2008; 9: 310.

27. Huang C-H, Su M-G, Kao H-J, Jhong J-H, Weng S-L, Lee T-Y. UbiSite: incorporating two-layered machine learning method with substrate motifs to predict ubiquitin-conjugation site on lysines. BMC systems biology. 2016; 10 (suppl 1): S6.

28. Shao J, Xu D, Hu L, Kwan Y-W, Wang Y, Kong X, et al. Systematic analysis of human lysine acetylation proteins and accurate prediction of human lysine acetylation through bi-relative adapted binomial score Bayes feature representation. Molecular BioSystems. 2012; 8: 2964-73.

29. Fan W, Xu X, Shen Y, Feng H, Li A, Wang M. Prediction of protein kinase-specific phosphorylation sites in hierarchical structure using functional information and random forest. Amino acids. 2014; 46: 1069-78.

30. Du Y, Zhai Z, Li Y, Lu M, Cai T, Zhou B, et al. Prediction of Protein Lysine Acylation by Integrating Primary Sequence Information with Multiple Functional Features. Journal of proteome research. 2016; 15: 4234-44. 
31. Li T, Du P, Xu N. Identifying human kinase-specific protein phosphorylation sites by integrating heterogeneous information from various sources. PloS one. 2010; 5: e15411.

32. Wang B, Wang M, Li A. Prediction of post-translational modification sites using multiple kernel support vector machine. PeerJ. 2017; 5: e3261.

33. Minguez P, Parca L, Diella F, Mende DR, Kumar R, Helmer-Citterich M, et al. Deciphering a global network of functionally associated post-translational modifications. Molecular systems biology. 2012; 8: 599.

34. Beltrao P, Albanèse V, Kenner LR, Swaney DL, Burlingame A, Villén J, et al. Systematic functional prioritization of protein posttranslational modifications. Cell. 2012; 150: 413-25.

35. Schweiger R, Linial M. Cooperativity within proximal phosphorylation sites is revealed from large-scale proteomics data. Biology direct. 2010; 5: 6 .

36. Minguez P, Letunic I, Parca L, Bork P. PTMcode: a database of known and predicted functional associations between post-translational modifications in proteins. Nucleic acids research. 2013; 41: D306-D11.

37. Lu Z, Cheng Z, Zhao Y, Volchenboum SL. Bioinformatic analysis and post-translational modification crosstalk prediction of lysine acetylation. PloS one. 2011; 6: e28228.

38. Kuo C-Y, Shieh C, Cai F, Ann DK. Coordinate to guard: crosstalk of phosphorylation, sumoylation, and ubiquitylation in DNA damage response. Frontiers in oncology. 2012; 1: 61.

39. Wimmer P, Blanchette P, Schreiner S, Ching W, Groitl P, Berscheminski J, et al. Cross-talk between phosphorylation and SUMOylation regulates transforming activities of an adenoviral oncoprotein. Oncogene. 2013; 32: 1626-37.

40. Hart GW, Slawson C, Ramirez-Correa G, Lagerlof O. Cross talk between O-GlcNAcylation and phosphorylation: roles in signaling, transcription, and chronic disease. Annual review of biochemistry. 2011; 80: 825-58.

41. Yao H, Li A, Wang M. Systematic analysis and prediction of in situ cross talk of O-GlcNAcylation and phosphorylation. BioMed research international. 2015; 2015.

42. Cheung P, Tanner KG, Cheung WL, Sassone-Corsi P, Denu JM, Allis CD. Synergistic coupling of histone $\mathrm{H} 3$ phosphorylation and acetylation in response to epidermal growth factor stimulation. Molecular cell. 2000; 5: 905-15.

43. Yang X-J, Seto E. Lysine acetylation: codified crosstalk with other posttranslational modifications. Molecular cell. 2008; 31: 449-61.

44. Cook C, Carlomagno Y, Gendron TF, Dunmore J, Scheffel K, Stetler C, et al. Acetylation of the KXGS motifs in tau is a critical determinant in modulation of tau aggregation and clearance. Human molecular genetics. 2013; 23: 104-16.

45. Daujat S, Bauer U-M, Shah V, Turner B, Berger S, Kouzarides T. Crosstalk between CARM1 methylation and CBP acetylation on histone H3. Current Biology. 2002; 12: 2090-7.

46. $\mathrm{Xu} \mathrm{L}$, Chen J, Gao J, Yu H, Yang P. Crosstalk of homocysteinylation, methylation and acetylation on histone H3. Analyst. 2015; 140: 3057-63.

47. Kurash JK, Lei H, Shen Q, Marston WL, Granda BW, Fan H, et al. Methylation of p53 by Set7/ 9 mediates p53 acetylation and activity in vivo. Molecular cell. 2008; 29: 392-400.

48. Parker BL, Shepherd NE, Trefely S, Hoffman NJ, White MY, Engholm-Keller $\mathrm{K}$, et al. Structural basis for phosphorylation and lysine acetylation cross-talk in a kinase motif associated with myocardial ischemia and cardioprotection. Journal of Biological Chemistry. 2014; 289: 25890-906.

49. Pan Z, Liu Z, Cheng H, Wang Y, Gao T, Ullah S, et al. Systematic analysis of the in situ crosstalk of tyrosine modifications reveals no additional natural selection on multiply modified residues. Scientific reports. 2014; 4: 7331.

50. Wang M, Jiang Y, Xu X. A novel method for predicting post-translational modifications on serine and threonine sites by using site-modification network profiles. Molecular BioSystems. 2015; 11: 3092-100.

51. Lu CT, Huang KY, Su MG, Lee TY, Bretana NA, Chang WC, et al. DbPTM 3.0: an informative resource for investigating substrate site specificity and functional association of protein post-translational modifications. Nucleic acids research. 2013; 41: D295-305.

52. Li H, Xing X, Ding G, Li Q, Wang C, Xie L, et al. SysPTM: a systematic resource for proteomic research on post-translational modifications. Molecular \& Cellular Proteomics. 2009; 8: 1839-49.

53. Diella F, Cameron S, Gemünd C, Linding R, Via A, Kuster B, et al. Phospho. ELM: a database of experimentally verified phosphorylation sites in eukaryotic proteins. BMC bioinformatics. 2004; 5: 79

54. Peri S, Navarro JD, Kristiansen TZ, Amanchy R, Surendranath V, Muthusamy $B$, et al. Human protein reference database as a discovery resource for proteomics. Nucleic acids research. 2004; 32: D497-D501.

55. Hornbeck PV, Kornhauser JM, Tkachev S, Zhang B, Skrzypek E, Murray B, et al. PhosphoSitePlus: a comprehensive resource for investigating the structure and function of experimentally determined post-translational modifications in man and mouse. Nucleic acids research. 2012; 40: D261-70.

56. Wang J, Torii M, Liu H, Hart GW, Hu Z-Z. dbOGAP-an integrated bioinformatics resource for protein O-GlcNAcylation. BMC bioinformatics. 2011; 12: 91.

57. Huang Y, Niu B, Gao Y, Fu L, Li W. CD-HIT Suite: a web server for clustering and comparing biological sequences. Bioinformatics. 2010; 26: 680-2.

58. Zhao X, Zhang $\mathrm{W}, \mathrm{Xu}$ X, Ma Z, Yin M. Prediction of protein phosphorylation sites by using the composition of k-spaced amino acid pairs. PloS one. 2012; 7: e46302.
59. Chen Z, Chen Y-Z, Wang X-F, Wang C, Yan R-X, Zhang Z. Prediction of ubiquitination sites by using the composition of k-spaced amino acid pairs. PloS one. 2011; 6: e22930.

60. Neuberger G, Schneider G, Eisenhaber F. pkaPS: prediction of protein kinase A phosphorylation sites with the simplified kinase-substrate binding model. Biology direct. 2007; 2 : 1 .

61. Li F, Li C, Wang M, Webb GI, Zhang Y, Whisstock JC, et al. GlycoMine: a machine learning-based approach for predicting $\mathrm{N}$-, C-and O-linked glycosylation in the human proteome. Bioinformatics. 2015; 31: 1411-9.

62. Gnad F, Ren S, Choudhary C, Cox J, Mann M. Predicting post-translational lysine acetylation using support vector machines. Bioinformatics. 2010; 26: 1666-8.

63. Hou T, Zheng G, Zhang P, Jia J, Li J, Xie L, et al. LAceP: lysine acetylation site prediction using logistic regression classifiers. PloS one. 2014; 9: e89575.

64. Wang J-R, Huang W-L, Tsai M-J, Hsu K-T, Huang H-L, Ho S-Y. ESA-UbiSite: accurate prediction of human ubiquitination sites by identifying a set of effective negatives. Bioinformatics. 2016; 33: 661-8.

65. Chang C-C, Lin C-J. LIBSVM: a library for support vector machines. ACM transactions on intelligent systems and technology (TIST). 2011; 2: 27.

66. Cui Y, Zhang M, Pestell R, Curran EM, Welshons WV, Fuqua SA. Phosphorylation of estrogen receptor a blocks its acetylation and regulates estrogen sensitivity. Cancer Research. 2004; 64: 9199-208.

67. Kassardjian A, Rizkallah R, Riman S, Renfro SH, Alexander KE, Hurt MM. The transcription factor $\mathrm{YY} 1$ is a novel substrate for Aurora B kinase at G2/M transition of the cell cycle. PloS one. 2012; 7: e50645.

68. Gu B, Zhu W-G. Surf the post-translational modification network of p53 regulation. International journal of biological sciences. 2012; 8: 672 .

69. Wang M, Wang T, Wang B, Liu Y, Li A. A Novel Phosphorylation Site-Kinase Network-Based Method for the Accurate Prediction of Kinase-Substrate Relationships. BioMed Research International. 2017; 2017:1826496. doi: $10.1155 / 2017 / 1826496$

70. Li A, Xue Y, Jin C, Wang M, Yao X. Prediction of N $\varepsilon$-acetylation on internal lysines implemented in Bayesian Discriminant Method. Biochemical and biophysical research communications. 2006; 350: 818-24.

71. $\mathrm{Xu} X$, Wang $\mathrm{M}$. Inferring Disease Associated Phosphorylation Sites via Random Walk on Multi-Layer Heterogeneous Network. IEEE/ACM Transactions on Computational Biology and Bioinformatics. 2016; 13: 836-44.

72. $\mathrm{Li} \mathrm{H}$, Wang $\mathrm{M}, \mathrm{Xu} \mathrm{X}$. Prediction of kinase-substrate relations based on heterogeneous networks. Journal of bioinformatics and computational biology. 2015; 13: 1542003.

73. Peng $\mathrm{C}, \mathrm{Li}$ A. A heterogeneous network based method for identifying GBM-related genes by integrating multi-dimensional data. IEEE/ACM transactions on computational biology and bioinformatics. 2017; 14: 713-20. 